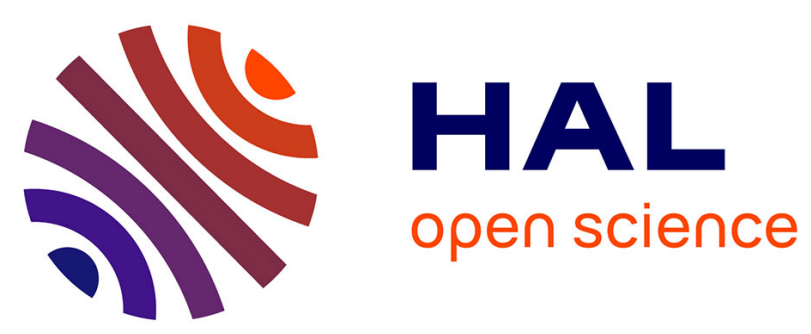

\title{
Datation radiocarbone directe et attribution culturelle des vestiges humains paléolithiques de la grotte d'Isturitz (Pyrénées-Atlantiques)
}

\author{
Dominique Gambier, Christian Normand, Jean-Marc Pétillon
}

\section{To cite this version:}

Dominique Gambier, Christian Normand, Jean-Marc Pétillon. Datation radiocarbone directe et attribution culturelle des vestiges humains paléolithiques de la grotte d'Isturitz (Pyrénées-Atlantiques).

Bulletin de la Société préhistorique française, 2013, 110 (4), pp.645-656. halshs-00913161

\section{HAL Id: halshs-00913161 \\ https://shs.hal.science/halshs-00913161}

Submitted on 3 Dec 2013

HAL is a multi-disciplinary open access archive for the deposit and dissemination of scientific research documents, whether they are published or not. The documents may come from teaching and research institutions in France or abroad, or from public or private research centers.
L'archive ouverte pluridisciplinaire HAL, est destinée au dépôt et à la diffusion de documents scientifiques de niveau recherche, publiés ou non, émanant des établissements d'enseignement et de recherche français ou étrangers, des laboratoires publics ou privés. 


\title{
Datation radiocarbone directe et attribution culturelle des vestiges humains paléolithiques de la grotte d'Isturitz (Pyrénées-Atlantiques)
}

\author{
Dominique Henry-Gambier, Christian Normand et Jean-Marc Pétillon
}

\begin{abstract}
Résumé : Les couches paléolithiques de la grotte d'Isturitz ont livré 119 vestiges humains, découverts lors des fouilles conduites par E. Passemard et R. et S. de Saint-Périer entre 1912 et 1959 . Très fragmentés et provenant à $90 \%$ de la tête osseuse, ces vestiges portent dans la grande majorité des cas des modifications anthropiques indiquant un même traitement du cadavre, en tout cas de la tête (décharnement, désarticulation, fracturation intentionnelle, parfois façonnage et/ou gravure des os). Ces restes humains présentent donc un intérêt majeur pour la connaissance des comportements mortuaires paléolithiques. Leur attribution culturelle est cependant problématique. Car même si $55 \%$ des pièces proviennent des couches du Magdalénien moyen (II, E, E $\omega, \mathrm{SI}$ ), une partie des pièces ont également été découvertes dans les autres couches du Paléolithique supérieur : Aurignacien (4\%), Gravettien (29\%), Solutréen (2\%), Magdalénien supérieur et Azilien ancien ( $8 \%$ ). Cette récurrence indique, soit la persistance d'un même comportement pendant toute la période, soit l'existence de mélanges stratigraphiques. Plusieurs indices nous font privilégier la seconde hypothèse : l'ancienneté des fouilles, la complexité de la stratigraphie et la difficulté à distinguer les différents niveaux, problèmes visibles à la lecture des publications d'E. Passemard et R. et S. de Saint-Périer, l'existence de raccords entre fragments provenant de couches différentes, et la mise en évidence de mélanges dans d'autres catégories de vestiges (industrie osseuse et art mobilier notamment). Dans le but de tester cette idée, des datations ${ }^{14} \mathrm{C}$ par SMA ont été effectuées sur un fragment de pariétal et un fragment de frontal humains de la couche II (Magdalénien moyen) et un fragment de pariétal humain de la couche III (Gravettien); les vestiges proviennent d'individus adultes et sont issus de trois individus différents. Les résultats indiquent qu'un des vestiges de la couche II et le vestige de la couche III se situent dans un intervalle correspondant au Magdalénien moyen : respectivement 14750 $\pm 50 \mathrm{BP}$, soit 18461-17652 cal. BP à 2 sigmas (GrA-45328), et $14640 \pm 50 \mathrm{BP}$, soit 18043-17540 cal. BP à 2 sigmas (GrA-45332). Ceci confirme l'hypothèse de la contamination de la couche III par la couche II. Le second vestige de la couche II est associé à un intervalle contemporain du Magdalénien supérieur : $13035 \pm 45$ BP, soit 16351-15174 cal. BP à 2 sigmas (GrA-45329). Ce résultat suggère un comportement pérenne par-delà la transition du Magdalénien moyen au Magdalénien supérieur, alors que les autres vestiges archéologiques indiquent que cette transition se caractérise par des changements importants : modification de la panoplie d'armature osseuses, du spectre des espèces chassées, des modalités d'approvisionnement en silex, des modalités de débitage lamellaire, de la fréquence et du type d'éléments de parure et de pièces d'art mobilier. Bien que d'autres datations soient nécessaires pour poursuivre la discussion, ces nouvelles dates confirment l'appartenance de la majorité des vestiges humains issus des fouilles anciennes au Magdalénien.
\end{abstract}

Mots clés : Paléolithique supérieur, Magdalénien moyen, Magdalénien supérieur, Isturitz, pratique mortuaire, datation ${ }^{14} \mathrm{C}$, Homo sapiens sapiens.

Abstract: This paper presents new AMS radiocarbon dates from three human bones housed at the Musée des Antiquités Nationales (Saint-Germain-en-Laye) and found during the Saint-Périer excavations in the Isturitz cave. This cave, located in the foothills of the western Pyrenees, is a major site of the Franco-Cantabrian region. It is well-known for having yielded a major Palaeolithic sequence with rich Magdalenian levels indicating long and intense occupation by human groups during the Upper Palaeolithic. The cave is divided into two main chambers: the Isturitz chamber (or Grande Salle) and the Saint-Martin chamber (fig. 1). Research was conducted in Isturitz by E. Passemard between 1912 and 1923, by R and S. de Saint-Périer between 1928 and 1948 (Passemard, 1924 and 1944; Saint-Périer, 1930 and 1936; Saint-Périer and Saint-Périer, 1952), and by C. Normand and others since 1999. The excavations of E. Passemard and R. and S. de Saint-Périer yielded an important assemblage of human remains from both adult and immature individuals. The cultural attribution of these bones is problematic, especially because of the complexity of the stratigraphy and the ancient date of the excavations (fig. 2); although the bones come from different levels of the sequence discovered in the 
cave, they are usually attributed to the Magdalenian (Gambier, 1990-1991). More than $80 \%$ of this assemblage consists of fragmented elements of the skull and mandible; anthropic modifications indicate a processing of corpses linked to either funerary or ritual, warlike practices (flesh removal, disarticulation, intentional breakage, sometimes shaping and engraving of the bones: Gambier, 1990-1991). While fragmentation limits the interest of these remains as regards the study of skeletal morphology, the anthropic modifications of the bones mean they are of major interest for understanding the mortuary behaviour of Upper Palaeolithic populations.

Three samples (fig. 3) were selected among the human remains found in the Grande Salle by R. and S. de Saint-Périer. Two are from layer II (Middle Magdalenian) and one is from layer III (Upper Gravettian). They include: IST II-231937, a fragment $(5.5 \mathrm{~cm} \times 6.4 \mathrm{~cm})$ of an adult parietal; IST II-41-1933, a fragment $(7.5 \mathrm{~cm} \times 6.5 \mathrm{~cm})$ of an adult frontal; and IST III-53-1937, a fragment of an adult parietal $(6.7 \mathrm{~cm} \times 6.0 \mathrm{~cm})$. These three fragments belong to three different adults. The bones were photographed and analysed prior to sampling. Sampling was done in areas without glue, consolidators, varnish or ink. They were submitted for AMS ${ }^{14} \mathrm{C}$ dating at Groningen (Centre for Isotope Research, Groningen University). The results indicate that the two bones 45328 IST II-23-1937 and 45332 IST III-53-1937 are dated respectively in the range of 18461-17652 and 18043-17540 cal. BP, corresponding to the Middle Magdalenian. The third, 45329 Isturitz II -41-1933, is associated with a range of 16351-15174 cal. BP, contemporary with the Upper Magdalenian.

The date obtained on the sample from layer III confirms the hypothesis of contamination of layer III, top of the Gravettian, by layer II, Middle Magdalenian. The assumption of the majority of human remains pertaining to the Middle Magdalenian is therefore admissible. The date obtained for the third sample is problematic because it falls within an interval corresponding to the Upper Magdalenian: it is close to the AMS date obtained on a fragment of antler barbed point ('harpoon head') from layer F1 (fig. 4: $13095 \pm 55$ BP or 16441-15223 cal. BP; Szmidt et al., 2009).

Like the others, this bone sample had anthropic modifications, and therefore the date could have been expected to integrate the interval corresponding to the Middle Magdalenian. Therefore, either the reliability of that date is uncertain - but no arguments support this hypothesis — or the processing of the corpse and head in the Upper Magdalenian was, if not identical, in any case, at least similar to that attested in the Middle Magdalenian.

Isolated human bones with anthropic modifications also exist in the Upper Magdalenian (Gambier, 1996) so this hypothesis cannot be ruled out. However, in the Grande Salle of Isturitz, the transition between Middle and Upper Magdalenian is visible in many categories of archaeological objects and fields of activity (flint and antler equipment, game hunted, ornaments and art: Pétillon, 2004 and 2006; Langlais, 2010; Laroulandie in Pétillon et al., to be published). These differences argue for major changes in human behaviour at the Middle-Upper Magdalenian transition. If the date on the human bone is reliable, it would mean that mortuary behaviour continued, at least partially, beyond these transformations.

Other dates are necessary to further the discussion and the dating programme on human bones should be continued. However, the assumption that the majority of human remains at Isturitz pertain to the Middle Magdalenian is reinforced by these new dates.

Keywords: Upper Palaeolithic, Middle Magdalenian, Upper Magdalenian, Isturitz, mortuary practice, ${ }^{14} \mathrm{C}$ dating, Homo sapiens sapiens.

$\mathrm{P}$ ARMI le matériel archéologique découvert dans la première moitié du $\mathrm{Xx}^{\mathrm{e}}$ siècle dans les niveaux paléolithiques de la grotte d'Isturitz, les vestiges humains constituent un ensemble important, comprenant plus d'une centaine de restes osseux et dentaires appartenant à des sujets immatures et adultes (tabl. 1). Leur attribution culturelle est problématique, et bien qu'issus, d'après leur marquage, de différents niveaux de la séquence représentée dans la grotte (Aurignacien à Azilien), ils sont généralement attribués au Magdalénien (Gambier, 1990-1991). Cet ensemble est composé de plus de $80 \%$ d'éléments fragmentés de la tête osseuse, et les os portent une série d'altérations qui indiquent un traitement des cadavres s'inscrivant soit dans le cadre de pratiques funéraires, soit dans un rituel en lien avec des pratiques guerrières (décharnement, désarticulation, fracturation intentionnelle, parfois façonnage et/ou gravure des os : Gambier, 1990-1991; Buisson et Gambier, 1991; Gambier, 1996). Si la fragmentation intense de ces restes limite leur intérêt pour la connaissance de la morphologie squelettique, les modifications anthropiques qu'ils présentent leur confèrent un intérêt majeur pour la compréhension des comportements mortuaires, d'autant que les vestiges humains en contexte magdalénien sont finalement assez rares et que les gestes funéraires sont peu connus. Les seules sépultures primaires indiscutables - Chancelade, Laugerie-Basse et Cap-Blanc en Dordogne, Saint-Germain-la-Rivière en Gironde et Lafaye dans le Tarn-et-Garonne - sont datées du Magdalénien moyen (Henry-Gambier, 2005). Les autres vestiges humains ne sont représentés que par des squelettes extrêmement fragmentaires ou des dents isolées et sont issus des différentes phases du Magdalénien; ils portent parfois, comme ceux d'Isturitz, des modifications anthropiques (Gambier, 1996).

Conservés au musée d'Archéologie nationale (MAN), à Saint-Germain-en-Laye, et à l'Institut de paléontologie humaine, à Paris, les vestiges humains d'Isturitz sont en cours de révision. Il s'agit de statuer à la fois sur leur contexte culturel et sur les comportements qu'ils suggèrent, pour tenter de mettre en évidence les changements de comportements devant la mort et les facteurs liés à 


\begin{tabular}{|c|c|c|c|c|c|}
\hline \multicolumn{6}{|l|}{ Collection Passemard } \\
\hline Attribution culturelle & Couche & Grande Salle & Salle de Saint-Martin & Salle inconnue & Total \\
\hline Azilien ancien & B & 1 & & & 1 \\
\hline Magdalénien moyen & $\mathrm{E}$ & 1 & & & 1 \\
\hline Magdalénien moyen & E omega & & 2 & & 2 \\
\hline Magdalénien moyen & E alpha & 2 & & & 2 \\
\hline Solutréen & F2 & 2 & & & 2 \\
\hline \multirow[t]{3}{*}{ Aurignacien } & A omega sud & & 1 & & 1 \\
\hline & Sans indication & & & 1 & 1 \\
\hline & Total & 6 & 3 & 1 & 10 \\
\hline \multicolumn{6}{|l|}{ Collection Saint-Périer } \\
\hline Attribution culturelle & Couche & Grande Salle & Salle de Saint-Martin & Salle inconnue & Total \\
\hline Magdalénien supérieur et Azilien ancien & Ia & 1 & & & 1 \\
\hline Magdalénien supérieur & I & 7 & & & 7 \\
\hline Magdalénien moyen & SI & & 2 & & 2 \\
\hline Magdalénien moyen & II & 58 & & & 58 \\
\hline Gravettien & III & 23 & & & 23 \\
\hline Gravettien & IV & 12 & & & 12 \\
\hline Aurignacien & « Aurignacien » & & & 2 & 2 \\
\hline \multirow[t]{3}{*}{ Aurignacien } & V & 2 & & & 2 \\
\hline & Sans indication & & & 2 & 2 \\
\hline & Total & 103 & 2 & 4 & 108 \\
\hline Total des deux collections & & 109 & 5 & 5 & 119 \\
\hline
\end{tabular}

Tabl. 1 - Isturitz : localisation spatiale et stratigraphique des vestiges humains d'après le marquage des pièces. D'après Gambier (19901991), modifié après vérification des marquages et de l'inventaire par les auteurs.

Table 1 - Isturitz: spatial and stratigraphic provenance of human remains according to their labelling. After Gambier (1990-1991), modified after checking the labelling and cataloguing by the authors.

ces éventuelles évolutions. En effet, les modifications osseuses mise en évidence sur ces os interpellent dans la mesure où les pratiques funéraires indiscutables du Magdalénien ne sont connues qu'à travers quelques sépultures primaires, toutes situées plus au nord. Les gestes autour de défunts mis en évidence à Isturitz sont-ils une alternative à la sépulture primaire ou reflètent-ils des comportements moins pacifiques? En préalable à cette discussion, une évaluation fiable de leur position chronologique est essentielle.

Cet article présente les premières dates radiocarbone en SMA obtenues sur trois échantillons d'os humains de la collection Saint-Périer, et les attributions culturelles qui en découlent.

\section{LE SITE D'ISTURITZ : DESCRIPTION, HISTORIQUE ET STRATIGRAPHIE}

S te majeur de la Préhistoire franco-ibérique, situé au carrefour de quatre régions - Bassin aquitain, Pyrénées, haute vallée de l'Ëbre et corniche vasco-cantabrique -, la grotte d'Isturitz a livré une stratigraphie très longue débutant au Paléolithique moyen et couvrant la totalité du Paléolithique supérieur, témoignant d'une fréquentation longue et intense par les groupes humains. De dimensions imposantes, avec une surface totale de plus de $2500 \mathrm{~m}^{2}$ et une hauteur de plafond atteignant localement $15 \mathrm{~m}$, ce réseau correspond à l'une des cavités creusées par l'Arberoue dans la colline calcaire de Gaztelu (communes d'Isturitz et Saint-Martin-d'Arberoue, PyrénéesAtlantiques). Il est communément partagé en deux : la salle de Saint-Martin (ou salle Sud) et la Grande Salle ou salle d'Isturitz (ou encore salle Nord), auxquelles il faut ajouter deux salles « annexes », la salle des Rhinolophes et la salle des Phosphates (fig. 1).

Connue de tous temps, mentionnée dans des textes dont le plus ancien remonte à la fin du XVII ${ }^{e}$ siècle, la grotte fut exploitée comme gisement de phosphates entre 1895 et 1898 , ce qui entraîna la destruction presque totale des couches archéologiques de la salle des Phosphates (Boule, 1896). Elle fit ensuite l'objet de fouilles intensives par E. Passemard entre 1912 et 1923, fouilles poursuivies par R. et S. de Saint-Périer quasiment sans interruption entre 1928 et 1948, puis plus ponctuellement par S. de Saint-Périer seule jusqu'en 1959 (Passemard, 1924 et 1944; Saint-Périer, 1930 et 1936; Saint-Périer 


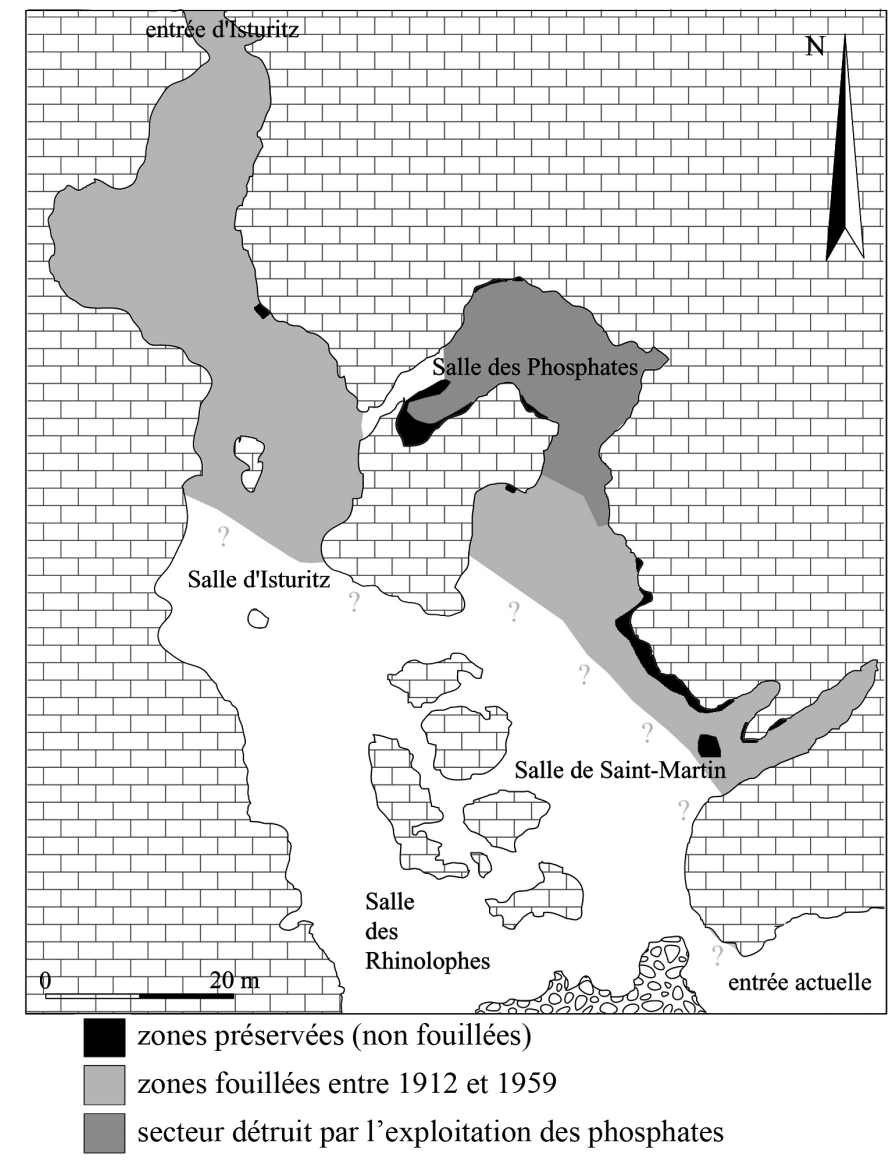

Fig. 1 - Isturitz : plan du site (document C. Normand).

Fig. 1 - Isturitz: map of the cave (document C. Normand).

et Saint-Périer, 1952). Entre 1996 et 1998, des opérations de sondage, d'observation de coupes et de tamisage de déblais, codirigées par A. Turq et l'un d'entre nous (C. N.), permirent d'évaluer le potentiel archéologique subsistant dans la grotte (Normand et Turq, 2007). Ces opérations débouchèrent en 1999 sur la mise en place d'une fouille programmée codirigée par I. Barandiarán, A. Cava, J. Fernandez Eraso et l'un d'entre nous (C. N.), puis, de 2000 à 2010, sur une seconde fouille placée sous la seule responsabilité de l'un d'entre nous (C. N.) et centrée sur les niveaux aurignaciens de la salle de SaintMartin (Normand, 2002 et 2005-2006; Normand et al., 2007; Szmidt et al., 2010). À partir des années 1990, l'industrie lithique, l'industrie osseuse, la faune et les vestiges anthropologiques des collections issues des fouilles anciennes firent l'objet de nombreuses études, souvent menées parallèlement à la reprise des fouilles et en relation avec la révision de la stratigraphie (Gambier, 19901991 ; Mujika, 1991 ; Esparza San Juan, 1995 ; Normand, 2002 et 2005-2006; Schwab, 2002 ; Foucher, 2004; Goutas, 2004; Pétillon, 2004 et 2006; Simonet, 2009; Langlais, 2010; Lacarrière et al., 2011).

À partir des données publiées par E. Passemard (1924 et 1944) et par R. de Saint-Périer (Saint-Périer, 1930 et 1936; Saint-Périer et Saint-Périer, 1952), H. Delporte (1974 et 1980-1981) établit une synthèse de la stratigra- phie des deux salles. Il proposa notamment un système de correspondances entre les différentes dénominations de couches utilisées par les fouilleurs successifs, en s'appuyant sur les premiers tableaux de corrélation publiés par R. et S. de Saint-Périer (Saint-Périer et Saint-Périer, 1952; Saint-Périer, 1965). La séquence retenue comportait, pour le Paléolithique : de l'Aurignacien, du Gravettien, du Solutréen, du Magdalénien et de l'Azilien dans la Grande Salle; du Moustérien, de l'Aurignacien et du Magdalénien dans la salle de Saint-Martin. Ces couches étaient surmontées dans les deux salles par des niveaux avec sépultures holocènes attribuées initialement à l'âge du Bronze (tabl. 2). Ce modèle stratigraphique, qui servit de référence durant les années 1980 et 1990, fut légèrement modifié par X. Esparza et J. A. Mujika (1996). Plus récemment, des études s'attachèrent à réviser la stratigraphie de l'Aurignacien de la salle de Saint-Martin (Normand et al., 2007), du Gravettien de la Grande Salle (Goutas, 2004; Simonet, 2009) et du Magdalénien de la Grande Salle (Pétillon, 2004 et 2006). Ces recherches récentes confirmèrent que les principales phases d'occupation du site avaient bien été correctement identifiées par les fouilleurs de la première moitié du $\mathrm{Xx}^{\mathrm{e}}$ siècle. Elles mirent cependant en évidence, dans la salle de Saint-Martin, des subdivisions stratigraphiques beaucoup plus fines que celles qui avaient été observées par ces 


\begin{tabular}{|c|c|c|c|c|c|}
\hline \multicolumn{3}{|c|}{ Couches de la Grande Salle } & \multicolumn{3}{|c|}{ Couches de la Salle de Saint-Martin } \\
\hline Passemard & Saint-Périer & Attribution & Passemard & Saint-Périer & Attribution \\
\hline stalagmite & & sépultures âge du Bronze & stalagmite & & sépultures âge du Bronze \\
\hline $\mathrm{B}$ & Ia & Magdalénien final et Azilien & \multirow{2}{*}{\multicolumn{3}{|c|}{ (pas de couches correspondantes) }} \\
\hline F1 & I & Magdalénien supérieur & & & \\
\hline $\mathrm{E}$ & II & Magdalénien moyen & E oméga & SI & Magdalénien moyen \\
\hline argile & IIIa (sommet) & stérile & \multirow{4}{*}{\multicolumn{3}{|c|}{ (pas de couches correspondantes) }} \\
\hline $\mathrm{F} 2$ & IIIa & Solutréen & & & \\
\hline argile & IIIlb & traces de Solutréen & & & \\
\hline $\mathrm{C}$ & III & « Aurignacien supérieur » (Gravettien) & & & \\
\hline F3 & IV & «Aurignacien supérieur » (Gravettien) & $\mathrm{x}, \mathrm{y}$ & SII & Aurignacien ou Gravettien? \\
\hline blocs & V (sommet) & stérile & blocs & argile & stérile \\
\hline A & $\mathrm{V}$ & Aurignacien & A & SIII & Aurignacien \\
\hline \multirow{3}{*}{\multicolumn{3}{|c|}{ (pas de couches correspondantes) }} & M & SIV & Moustérien \\
\hline & & & \multicolumn{3}{|c|}{ repaire d'ours } \\
\hline & & & $\mathrm{P}$ & SV & Moustérien \\
\hline \multicolumn{2}{|c|}{ stalagmite } & stérile & \multicolumn{2}{|c|}{ stalagmite } & stérile \\
\hline \multicolumn{2}{|c|}{ argiles, sables, graviers } & stérile & \multicolumn{2}{|c|}{ argiles, sables, graviers } & stérile \\
\hline
\end{tabular}

Tabl. 2 - Isturitz : équivalences entre les stratigraphies Passemard et de Saint-Périer (d'après Delporte, 1974 et 1980-1981). Nous avons rectifié, dans ce tableau, l'emplacement du sous-niveau IIIb, qu'H. Delporte plaçait au-dessus du IIIa alors qu'il lui est sousjacent (Saint-Périer et Saint-Périer, 1952, p. 18).

Table 2 - Isturitz: equivalence between the Passemard and de Saint-Périer stratigraphies (after Delporte, 1974 and 1980-1981). In this table we have corrected the position of sub-level IIIb, placed above IIIa by H. Delporte although it is actually under it (Saint-Périer and Saint-Périer, 1952, p. 18).

fouilleurs; ainsi que, dans les deux salles, des problèmes d'attribution stratigraphique d'une partie du matériel des collections anciennes. Ces résultats étaient bien sûr prévisibles étant donné l'ampleur du site, la grande complexité de son remplissage, les conditions de fouille et les techniques de terrain en usage à l'époque, la longue histoire des collections après la fouille (aléas de la conservation, du marquage des pièces...), etc. Les vestiges humains n'échappent pas à ces problèmes.

\section{ORIGINE DES VESTIGES HUMAINS}

D' après leur marquage, la majorité de ces vestiges (92\%) provient des fouilles Saint-Périer. Quatrevingt-onze pour cent ont été trouvés dans la Grande Salle, $4 \%$ dans la salle de Saint-Martin et $4 \%$ n'ont pu être localisés (tabl. 1) ${ }^{1}$. Ils se répartissaient dans toute la séquence du Paléolithique supérieur, mais $55 \%$ proviennent du Magdalénien moyen (niveaux II, E, E $\omega$, SI; tabl. 1).

Quatre-vingt-dix pour cent des vestiges appartiennent à la tête osseuse (tabl. 3); 74\% de ces vestiges crâniens ont subi une fragmentation et des altérations anthropiques de même type, indiquant une chaîne identique de traitement de la tête. Cette homogénéité, quelle que soit l'origine stratigraphique annoncée, conduit à deux hypothèses : soit la pérennité d'une même pratique de l'Aurignacien au Magdalénien supérieur, soit l'appartenance à un seul ensemble culturel et, donc, l'existence de mélanges lors des fouilles. Plusieurs éléments nous font privilégier la seconde hypothèse et nous amènent à discuter la fiabilité d'une partie des attributions stratigraphiques :

1) Un réexamen, par les auteurs de cet article, des vestiges conservés au MAN a permis de réattribuer, sur des critères de patine et de texture ainsi que sur la présence de concrétion, trois os du squelette infracrânien aux sépultures holocènes ${ }^{2}$ implantées près de l'entrée actuelle. Il faut d'ailleurs souligner que ces os, contrairement aux autres, étaient complets et ne présentaient pas de modification.

\begin{tabular}{|l|c|c|} 
Origine anatomique & $\mathrm{N}$ & $\begin{array}{c}\text { N avec modifications } \\
\text { anthropiques }\end{array}$ \\
\hline Crâne & 74 & 55 \\
\hline Mandibule & 14 & 10 \\
\hline Squelette infracrânien & 13 & 4 \\
\hline Dent isolée & 18 & 0 \\
\hline Total & 119 & 69 \\
soit $68 \%$ des restes osseux
\end{tabular}

Tabl. 3 - Isturitz : nombre de vestiges humains présentant des modifications d'origine anthropique.

Table 3 - Isturitz: number of human remains with anthropic modifications. 


\begin{tabular}{|c|l|l|c|l|}
\hline $\mathrm{N}^{\circ}$ os & Marquage pièce & & $\mathrm{N}^{\circ}$ os & Marquage pièce \\
\hline 6 & IST III 1928 & avec & 52 & IST II 1930 \\
\hline 7 & IST II 1931 & avec & 8 & IST II 1931 \\
\hline 12 & IST II 1932 & avec & 20 & IST II 1932 \\
\hline 21 & IST II 1931 & avec & 43 & IST II 1931 \\
\hline 29 & IST III 1937 & avec & 53 & IST III 1937 \\
\hline 37 & IST II 1930 & avec & 98 & F1 Beta \\
\hline 50 & IST II 1931 & avec & 79 & E Alpha 1914 \\
\hline 72 & IST II 1934 & avec & 99 & IST I 1933 \\
\hline
\end{tabular}

Tabl. 4 - Isturitz : raccords effectués entre les vestiges humains (Gambier, 1990-1991).

Table 4 - Isturitz: connections made between the human remains (Gambier, 1990-1991).

\begin{tabular}{|l|c|c|}
\hline Grande Salle & $\mathrm{N}$ & $\%$ \\
\hline Magdalénien supérieur et Azilien ancien & 9 & $8 \%$ \\
\hline Magdalénien moyen & 61 & $56 \%$ \\
\hline Solutréen & 2 & $2 \%$ \\
\hline Gravettien (sommet) & 23 & $21 \%$ \\
\hline Gravettien (base) & 12 & $11 \%$ \\
\hline Aurignacien & 2 & $2 \%$ \\
\hline Total & 109 & $100 \%$ \\
\hline Salle de Saint-Martin & $\mathrm{N}$ & $\%$ \\
\hline Magdalénien moyen & 4 & $80 \%$ \\
\hline Aurignacien & 1 & $20 \%$ \\
\hline Total & 5 & $100 \%$ \\
\hline
\end{tabular}

Tabl. 5 - Isturitz : attribution culturelle des vestiges humains d'après leur marquage.

Table 5 - Isturitz: cultural attribution of the human remains according to their labelling.

2) Des raccords entre fragments de crâne humain issus de couches différentes ont été effectués (tabl. 4), notamment dans la Grande Salle entre les couches II et I (respectivement Magdalénien moyen et supérieur), et entre les couches III et II (respectivement Gravettien et Magdalénien moyen), fouilles R. et S. de Saint-Périer; mais aussi entre la couche II (Magdalénien moyen), fouilles $\mathrm{R}$. et $\mathrm{S}$. de Saint-Périer, et la couche F1 (Magdalénien supérieur), fouilles E. Passemard.

3) Le mélange entre couches est, en outre, attesté par d'autres vestiges archéologiques, notamment de l'industrie osseuse et de l'art mobilier (Gambier 1990-1991; Pétillon, 2004; Goutas, 2004).

4) Des contradictions entre l'origine stratigraphique mentionnée dans certaines publications et celle indiquée sur le marquage des objets peuvent aussi être relevées, témoignant d'une certaine confusion.

La question posée est donc la suivante : à quel ensemble culturel rattacher les vestiges humains (tabl. 5)? Dans la salle de Saint-Martin, quatre restes sur cinq proviendraient du Magdalénien moyen (couches E $\omega$ et SI). Dans la Grande Salle, 61 vestiges sur 109 seraient aussi issus des couches attribuées à cette culture (couches E et II). Mais, dans cette même salle, 34 autres pièces portent un marquage qui renvoie aux couches immédiatement sus-jacentes -9 restes dans les couches $\mathrm{I}^{\mathrm{a}}$, I et B, Magdalénien supérieur et probable Azilien ancien (Langlais, 2010) - et sous-jacentes - couche F2, Solutréen (2 restes); couche III, sommet du Gravettien (23 restes). Pour ces 34 vestiges, l'hypothèse de mélanges à l'interface Magdalénien moyen-Magdalénien supérieur d'une part et à l'interface Gravettien-Solutréen-Magdalénien moyen d'autre part doit être envisagée, malgré le soin constant pris par les fouilleurs pour distinguer précisément les différentes couches.

Cette hypothèse est soutenue par une analyse critique de la stratigraphie magdalénienne de la Grande Salle qui a montré, en s'appuyant notamment sur la répartition par couche des types d'armatures osseuses, l'existence de certaines erreurs d'attribution du matériel (Pétillon, 2004). Il semble en effet que, à la suite de lectures stratigraphiques légèrement divergentes, E. Passemard a intégré une partie des vestiges du Magdalénien supérieur dans la couche attribuée au Magdalénien moyen, tandis que R. de Saint-Périer aurait, à l'inverse, inclus certaines pièces du Magdalénien moyen dans la couche attribuée au Magdalénien supérieur. Cette situation n'a rien d'étonnant si l'on considère la description de R. de Saint-Périer : «Si nos fouilles nous ont révélé partout l'existence des deux niveaux [I, Magdalénien supérieur, et II, Magdalénien moyen], il s'en faut que leur superposition ait été régulière. L'un et l'autre ondulaient presque sans cesse et leurs sinuosités, s'insinuant en tous sens, enchâssaient ici le niveau I dans le II et là, le II dans le I. La différence très nette de leur aspect physique permettait de les distinguer immédiatement [...] mais il y fallait une attention constante » (Saint-Périer, 1936, p. 8).

À la base du Magdalénien moyen, la distinction avec les niveaux solutréens semble également avoir été délicate, et il n'est pas surprenant que des confusions aient pu avoir lieu. R. de Saint-Périer indique ainsi que « tout le long des parois sur une largeur de 3 m environ et en face du piler gravé ", l'ensemble solutréen III ${ }^{a}$ se présentait comme une couche argileuse " aux limites incertaines, aux aspects variables, qui ne prenait un caractère défini que par la brusque apparition d'une de ces belles pièces entièrement couvertes de retouches $»$; à son sommet, la couche solutréenne « ne se discernait du Magdalénien que par la plus grande rareté des débris osseux » (Saint-Périer et Saint-Périer, 1952, p. 12). Comme l'a relevé N. Goutas (2004, p. 110), R. de Saint-Périer indique également qu'en plusieurs endroits des «poches magdaléniennes » s'insinuaient dans la couche solutréenne, ou inversement. De plus, ces irrégularités à l'interface entre couches II et III ${ }^{\mathrm{a}}$ sont figurées sur la coupe synthétique publiée en 1952 (Saint-Périer et Saint-Périer, 1952, fig. 4), mais sont absentes d'un premier dessin de la même coupe publié en 1936 (Saint-Périer, 1936, fig. 4; voir ici fig. 2); il est donc possible qu'elles n'aient été perçues qu'après coup, 


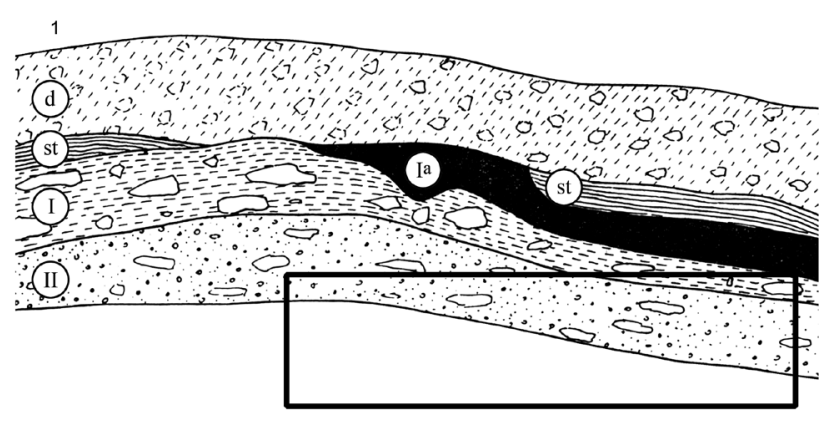

2

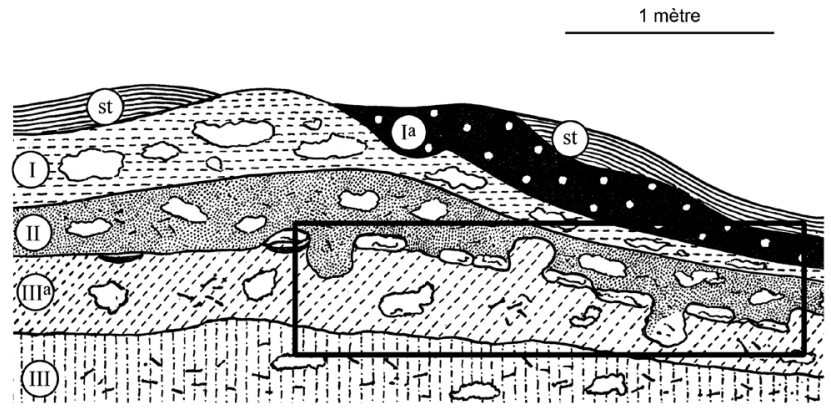

Fig. 2 - Isturitz : coupe stratigraphique de la Grande Salle « en avant du pilier, vers Isturitz ». 1 : détail du relevé publié in Saint-Périer, 1936; 2 : relevé publié in Saint-Périer et Saint-Périer, 1952, après enlèvement des déblais et fouille des niveaux inférieurs; $d$ : déblais; st : stalagmite. Encadré : les irrégularités (« poches ») à la base de la couche II ne figurent que sur la version de 1952.

Fig. 2 - Isturitz: stratigraphic profile of the Grande Salle "before the pillar, towards Isturitz". 1: detail of the version published in Saint-Périer, 1936; 2: version published in Saint-Périer and Saint-Périer, 1952, after removal of the backfill and excavation of the lower layers; $d$ : backfill; st: stalagmite. Framed area: the irregularities ('pockets') at the bottom of layer II are only visible on the 1952 version.

lors de la fouille des niveaux inférieurs, ce qui augmente bien sûr les risques d'erreur dans les attributions stratigraphiques.

Le fait que des éléments du Magdalénien moyen aient pu être attribués au Gravettien s'explique également par le fait que, toujours d'après les descriptions de R. de SaintPérier, la couche III - représentant archéologiquement le sommet de l'ensemble gravettien - était constituée de la même argile gris verdâtre que la couche III ${ }^{\mathrm{a}}$, se présentant seulement « avec beaucoup plus de continuité » (SaintPérier et Saint-Périer, 1952, p. 36). De fait, à l'issue d'une analyse critique portant, là encore, sur les types d'armatures osseuses, N. Goutas (2004, p. 106-117) a pu démontrer la présence dans la couche III d'une importante quantité de matériel du Solutréen et du Magdalénien moyen, y compris des types très caractéristiques comme les pointes de Lussac-Angles.

Un passage de R. de Saint-Périer, plusieurs fois cité pour illustrer les difficultés stratigraphiques d'Isturitz, résume bien les risques de contamination entre les couches du Magdalénien moyen et d'autres niveaux plus anciens ou plus récents : «Nous avons retrouvé à trois reprises différentes un lambeau d'une couche magdalénienne que nous avions attribué d'abord de par son aspect et sa place à un étage inférieur. La première fois c'était une poche de Magdalénien supérieur qui s'insinuait dans le Magdalénien ancien [...] Deux autres fois dans cette même salle, après notre publication, au cours d'une fouille austère dans l'Aurignacien, il nous a été donné de retrouver des lambeaux de la belle couche magdalénienne si riche en objets d'art » (Saint-Périer, 1947, p. 394-395).

Il faut ajouter que le réexamen de chaque reste humain conservé au MAN et supposé provenir des ensembles I et III a permis de constater, sur plusieurs vestiges, la présence d'un dépôt de sédiment brun-rouge qui évoque le sédiment de la couche II et confirme l'hypothèse de mélanges entre couches.
En revanche, comment expliquer les quatorze vestiges issus des couches IV (12), base du Gravettien, et $\mathrm{V}$ (2), Aurignacien, qui présentent pour une part une fragmentation et/ou des modifications osseuses comparables à celles observées sur le reste du matériel? La seule particularité de ce petit corpus est de comprendre quatre restes du squelette infracrânien, soit plus d'un tiers des vestiges infracrâniens collectés dans la cavité. Là aussi l'hypothèse d'un mélange entre couches, voire d'une intrusion du niveau II (Magdalénien moyen) dans les niveaux sous-jacents nous semble cependant la plus vraisemblable.

Cette hypothèse d'une appartenance des vestiges humains au Magdalénien moyen, récemment daté (Szmidt et al., 2009), restait à valider par la datation ${ }^{14} \mathrm{C}$ de certains d'entre eux. Une première série de datations par le radiocarbone en SMA sur trois fragments de crânes de la collection Saint-Périer (MAN) a donc été tentée.

\section{DATATION ${ }^{14} \mathrm{C}$ DE TROIS FRAGMENTS DE CRÂNE}

\section{Les échantillons}

Les trois échantillons (fig. 3) ont été choisis parmi les vestiges des fouilles de R. et S. de Saint-Périer dans la Grande Salle. Deux sont issus de la couche II, un provient de la couche III. Les os ont été photographiés et analysés avant le prélèvement. Ce dernier a été fait dans des zones sans colle, consolidant, vernis ni encre.

IST II-23-1937 : c'est un fragment $(5,5 \mathrm{~cm} \times 6,4 \mathrm{~cm})$ de pariétal, probablement droit, d'adulte dont une partie de la suture sagittale est préservée. Son épaisseur maximale est de $5 \mathrm{~mm}$. Il est limité par des cassures anciennes sans doute survenues sur os frais : elles sont encore incrustées d'un sédiment brun-rouge. L'état de la surface 


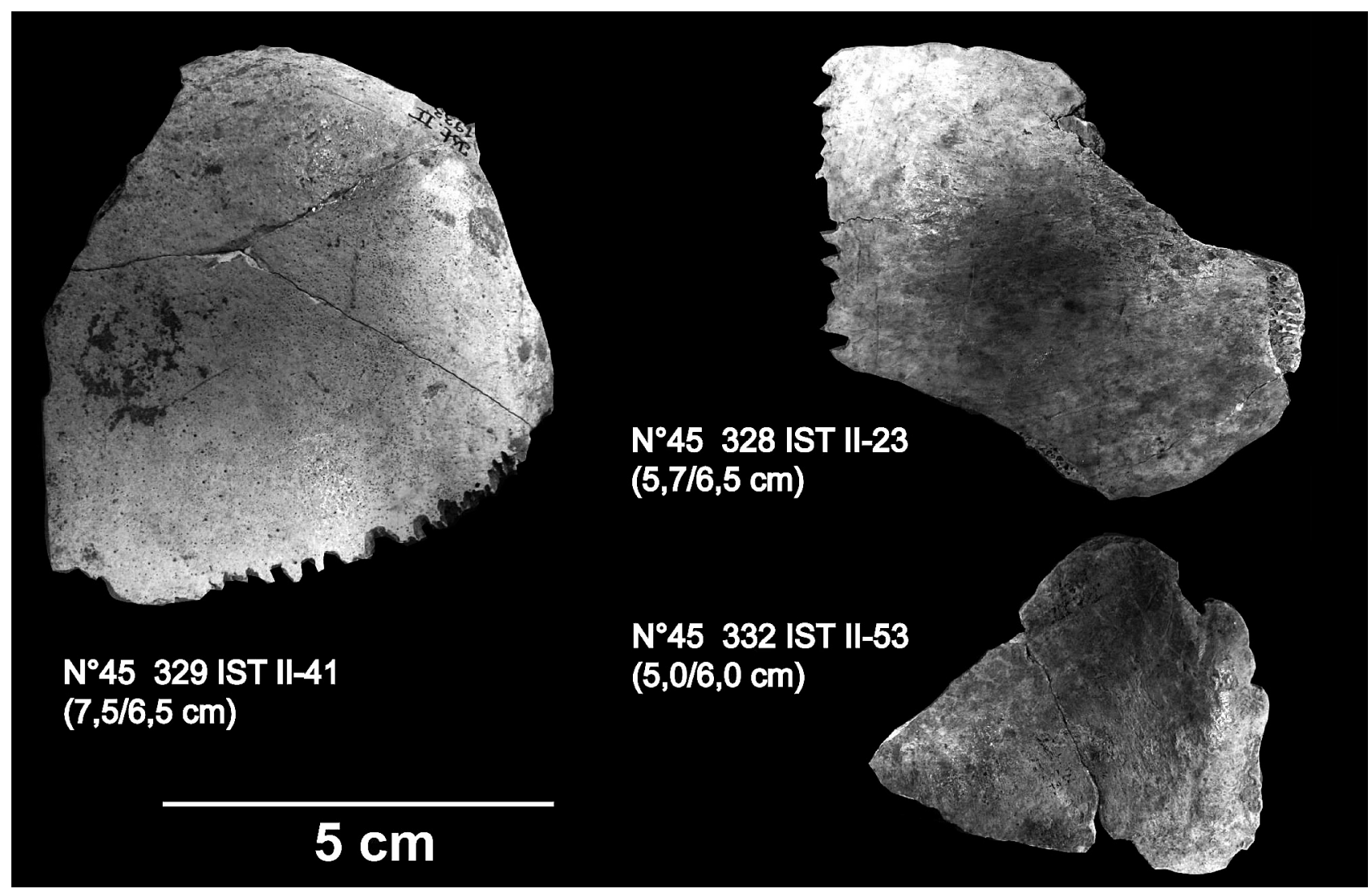

Fig. 3 - Isturitz : les trois fragments de crâne avant prélèvement pour datation. Collection Saint-Périer, MAN, Saint-Germain-en-Laye (cliché D. Henry-Gambier).

Fig. 3 - Isturitz: the three skull fragments before sampling for radiocarbon dating. Saint-Périer collection, MAN, Saint-Germain-enLaye (photo D. Henry-Gambier).

exocrânienne est très bon, et de nombreuses traces de raclage linéaires très fines peuvent être observées.

IST II-41-1933 : c'est un fragment $(7,5 \mathrm{~cm} \times 6,5 \mathrm{~cm})$ de frontal d'adulte restreint à la partie droite de l'écaille. Il est limité par la moitié droite de la suture coronale, des cassures anciennes (sagittale et transversale), peut-être sur os frais, et une cassure latérale récente. La surface exocrânienne est intacte, et, à la hauteur de la bosse frontale, on distingue une série de stries anthropiques (5 à $10 \mathrm{~mm}$ ) plus ou moins appuyées.

IST III-53-1937 : c'est un fragment de pariétal d'adulte $(6,7 \mathrm{~cm} \times 6,0 \mathrm{~cm})$ correspondant à l'angle antéro-inférieur du pariétal avec un petit segment de la suture coronale. Les cassures qui limitent ce fragment sont récentes. Son épaisseur maximale atteint $6,5 \mathrm{~mm}$. La surface exocrânienne est assez dégradée, et il est difficile d'être affirmatif quant à la présence de stries.

Ces fragments appartiennent à trois individus différents.

\section{Méthode et résultats}

Les os ont été datés au laboratoire de Groningen (Center for Isotope Research, Groningen University) et traités selon le protocole standard développé par ce laboratoire. Les mesures élémentaires et d'isotopes stables (tabl. 6) se situent toutes dans des marges normales. Les dates ont été calibrées par le programme CALIB version 6.0 selon intcal09.14C (Reimer et al., 2009). Aucune correction liée à l'effet réservoir marin n'a été appliquée. Les âges conventionnels obtenus placent les trois échantillons dans un intervalle qui correspond au Magdalénien. Par comparaison avec les dates obtenues sur des artefacts (Szmidt $e t$ al., 2009; ici fig. 4), l'échantillon 45328 IST II-23, qui se situe dans l'intervalle 18461-17652 cal. BP à 2 sigmas, appartient au Magdalénien moyen. C'est aussi le cas de l'échantillon 45332 IST-III-53 qui s'inscrit dans l'intervalle 18043-17540 cal. BP à 2 sigmas.

En revanche, l'échantillon 45329 IST II-41 se place entre 16351 et 15174 cal. BP à 2 sigmas, intervalle qui correspond au Magdalénien supérieur (fig. 4).

\section{DISCUSSION}

Ces résultats appellent plusieurs remarques :

1) La date obtenue sur l'échantillon de la couche III confirme l'hypothèse de la contamination de cette couche, sommet du Gravettien, par la couche II, Magdalénien moyen. L'hypothèse d'une appartenance d'une majorité des vestiges humains à la couche II et donc au Magdalénien moyen est donc recevable. 


\begin{tabular}{|l|l|l|l|}
\hline Échantillon & 45328 Isturitz II-23 & 45329 Isturitz II-41 & 45332 Isturitz III-53 \\
\hline Marquage & IST II 1937 & IST II 1933 & IST III 1937 \\
\hline Nature & os humain & os humain & os humain \\
\hline Os & pariétal & frontal & pariétal \\
\hline Salle & Grande Salle & Grande Salle & Grande Salle \\
\hline Fouille & Saint-Périer & Saint-Périer & Saint-Périer \\
\hline Couche & II & II & III \\
\hline Phase culturelle & Magdalénien moyen & Magdalénien moyen & Gravettien \\
\hline Âge conventionnel (BP) & $14750 \pm 50$ & $13035 \pm 45$ & $14640 \pm 50$ \\
\hline Âge calibré (BP) à 2 sigmas et probabilité & $18461-17652(\mathrm{p}=1)$ & $16351-15174(\mathrm{p}=1)$ & $18043-17540(\mathrm{p}=1)$ \\
\hline Laboratoire & GrA-45328 - IST 2-23 & GrA-45329 -IST 2-41 & GrA -45332 IST 3-53 \\
\hline Masse utilisée (mg) & 2916 & 1453 & 5430 \\
\hline Masse de collagène (mg) & 186,0 & 31,5 & 146,3 \\
\hline $15 \delta(\%)$ & 12,17 & 14,14 & 14,14 \\
\hline$\%$ C & 44,2 & 42,5 & 45,5 \\
\hline$\%$ N & 16,1 & 14,6 & 16,1 \\
\hline C/N & 2,7 & 2,9 & 2,8 \\
\hline C/N atomique & 3,2 & 3,4 & 3,3 \\
\hline
\end{tabular}

Tabl. 6 - Isturitz : datation ${ }^{14} \mathrm{C}$ de trois échantillons d'os humain. Calibration : CALIB version 6.0; intcal09.14C (Reimer et al., 2009). Table 6 - Isturitz: ${ }^{14}$ C dating of three samples of human bone. Calibration: CALIB 6.0; intcal09.14C (Reimer et al., 2009).

2) La date obtenue pour le troisième échantillon pose un problème, car elle se place dans un intervalle qui correspond au Magdalénien supérieur : elle est extrêmement proche de la date ${ }^{14} \mathrm{C}$ par SMA obtenue sur un fragment de pointe à une rangée de barbelures (« harpon ») de la couche F1 (Szmidt et al., 2009 : respectivement 13035 $\pm 45 \mathrm{BP}$, soit 16351-15174 cal. BP à 2 sigmas, et 13095 $\pm 55 \mathrm{BP}$, soit 16441-15223 cal. BP à 2 sigmas). De fait, à Isturitz, la date de $13455 \pm 55 \mathrm{BP}$ (soit 16886-16291 cal. BP à 2 sigmas) obtenue sur une pièce caractéristique de la couche du Magdalénien supérieur (une pointe à base fourchue) et la date de $13605 \pm 65 \mathrm{BP}$ (soit 16958-16528 cal. BP à 2 sigmas) obtenue sur une pièce caractéristique de la couche du Magdalénien moyen (baguette demironde à décor de tubercules) semblent indiquer que la transition entre Magdalénien moyen et supérieur se place aux alentours de 17000-16500 cal. BP. Cette hypothèse est cohérente avec le cadre chronologique élaboré à partir des dates ${ }^{14} \mathrm{C}$ par SMA obtenues sur les niveaux du Magdalénien moyen et supérieur dans de nombreux sites d'Espagne et du Sud de la France (Langlais, 2010, p. 284285 et 292-293). Plusieurs dates du Magdalénien supérieur se situent en effet dans l'intervalle 17000-16000 cal. BP, tandis que les dates du Magdalénien moyen ne sont pas plus tardives que ce même intervalle. Dans cette perspective, la date GrA-45329 obtenue sur l'échantillon de la couche II se situe sans ambiguïté dans la fourchette du Magdalénien supérieur. L'os humain dont provient cet échantillon portait, comme les autres, des modifications osseuses, et, pour cette raison, la date attendue aurait dû se placer dans l'intervalle correspondant au Magdalénien moyen. Il faut donc soit admettre que la fiabilité de cette date est douteuse - or aucun argument n'appuie cette hypothèse -, soit supposer un traitement du cadavre et de la tête osseuse au Magdalénien supérieur qui soit sinon identique en tout cas proche de celui mis en évidence au Magdalénien moyen. Des vestiges isolés avec modifications anthropiques existent aussi au Magdalénien supérieur (Gambier, 1996), aussi cette hypothèse ne peut être totalement écartée. Mais il se trouve par ailleurs que, dans la Grande Salle d'Isturitz, la transition entre Magdalénien moyen et supérieur est visible à travers de nombreuses catégories de vestiges archéologiques. Cette transition est en effet marquée par un changement dans les panoplies d'armatures osseuses (Pétillon, 2004 et 2006). Le spectre des espèces chassées se modifie également, avec une intensification de l'exploitation des oiseaux (Laroulandie in Pétillon et al., à paraître). Dans l'industrie lithique, certaines modalités de débitage lamellaire (« sur tranche transversale sur encoche ») ne sont attestées que dans le Magdalénien moyen (Langlais, 2010). Les modalités d'approvisionnement en silex changent également, et la proportion des matériaux allochtones baisse assez sensiblement au Magdalénien supérieur (C. N., étude en cours; Langlais, 2010). Enfin, toujours dans le Magdalénien supérieur, le nombre de dents aménagées en éléments de parure diminue fortement, tandis que la décoration des objets se raréfie et s'appauvrit; dans ces deux domaines, le répertoire des espèces utilisées se restreint également, puisque bovinés et carnivores disparaissent à la fois de l'art mobilier et de la parure, où ils étaient bien représentés durant le Magdalénien moyen (Pétillon, 2004). Il s'agit de différences fortes qui, ajoutées à d'autres, plaident en faveur d'importantes modifications de comportements au moment de la transition Magdalénien moyen-Magdalénien supérieur. Si la date obtenue 


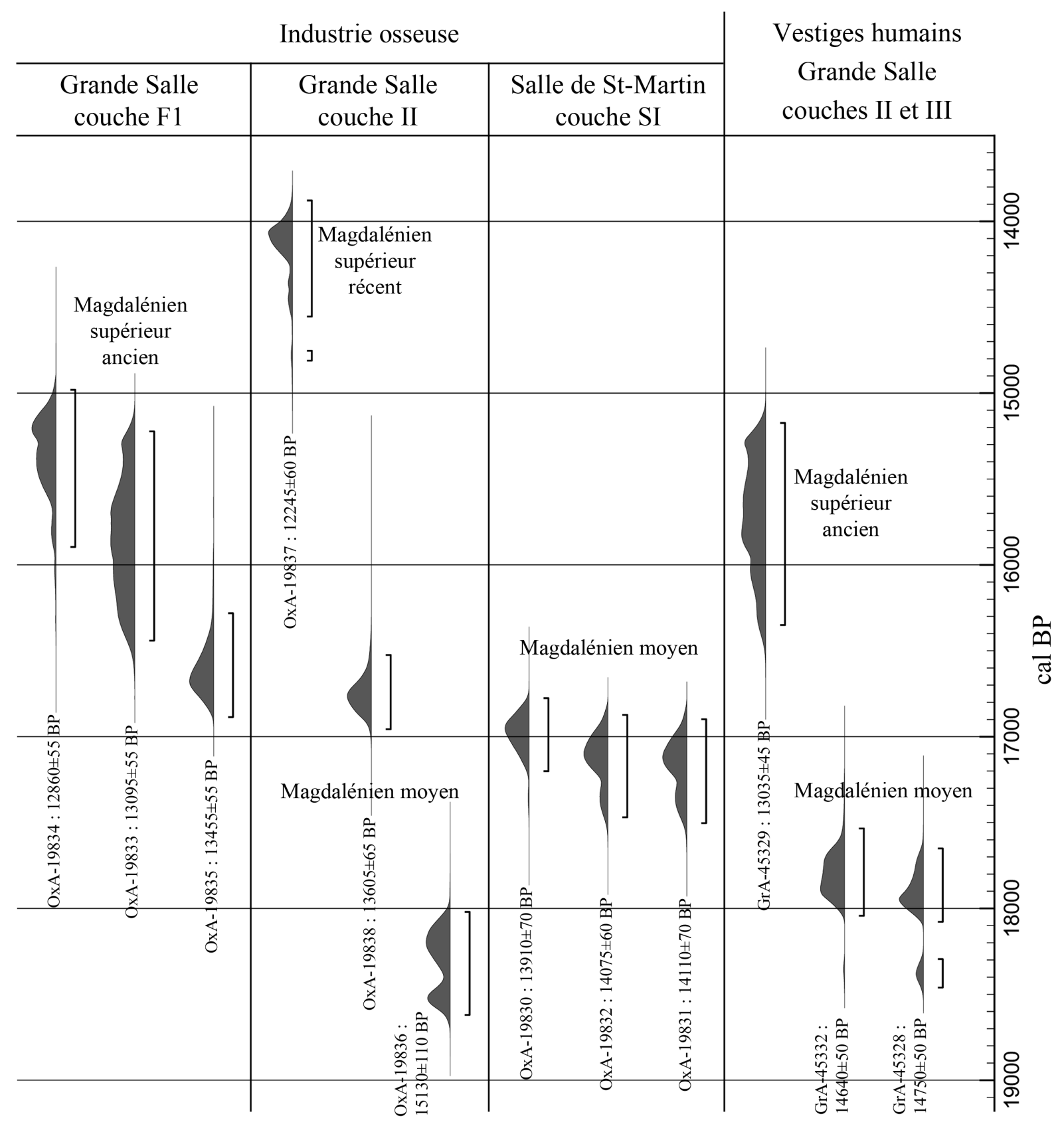

Fig. 4 - Isturitz : comparaison entre les dates obtenues sur les pièces d'industrie osseuse et celles obtenus sur les fragments de crânes humains. Les crochets indiquent l'intervalle de confiance à 2 sigma. Dates des objets osseux d'après Szmidt et al., 2009; graphique réalisé avec OxCal 4.1; intca109.14C (Reimer et al., 2009).

Fig. 4 - Isturitz: comparison between the radiocarbon dates obtained on osseous artefacts and on fragments of human skulls. Brackets show the 2-sigma interval. Dates on osseous artefacts after Szmidt et al., 2009; graph made with OxCal 4.1 - intcal09.14C (Reimer et al., 2009).

sur le fragment d'os humain est fiable, cela signifierait que les comportements mortuaires échappent en partie à ces évolutions.

Il est pour l'instant difficile d'aller au-delà dans la discussion avec seulement trois datations. Il est nécessaire de poursuivre le programme de datation, en particulier de vestiges issus, d'après leur marquage, des niveaux I, IV et $\mathrm{V}$.

Il reste que l'hypothèse d'une appartenance de la majorité des vestiges humains d'Isturitz au Magdalénien moyen est renforcée par ces trois nouvelles datations ${ }^{14} \mathrm{C}$ sur os humain.

Remerciements : Nous remercions C. Schwab, conservatrice au musée d'Archéologie nationale, à Saint-Germain-en-Laye, qui nous a autorisés à prélever des échantillons pour datation, et J. Darricau, propriétaire du site d'Isturitz, qui accueille les fouilles. Ces recherches ont bénéficié du soutien du CNRS et des projets ANR Guerope (dir. L. Baray) et Magdatis (dir. J.-M. Pétillon, projet ANR 2011 BSH3 0005). 


\section{NOTES}

(1) Lors du tri de la faune, effectué par D. Henry-Gambier dans les collections du MAN et par D. Henry-Gambier et A. Faucheux dans celles d'Hasparren, une vingtaine de fragments humains ont été identifiés. Ils ne sont pas intégrés dans cet article, car leur étude est en cours. Plusieurs, voire la majorité, sont très probablement rattachables aux sépultures holocènes de la salle de Saint-Martin (voir note 2; P. Cour- taud, étude en cours). Quoi qu'il en soit, ils ne modifient ni le profil, ni les caractéristiques de l'assemblage, ni les questionnements. En outre, les publications signalent la découverte au XIX ${ }^{\mathrm{e}}$ siècle de deux mandibules aujourd'hui perdues. Il faut d'ailleurs signaler que certains vestiges de la collection Saint-Périer restent introuvables.

(2) L'étude du matériel réalisée à l'occasion d'une reprise des recherches sur la zone funéraire proche de l'entrée de SaintMartin attribue désormais tout ou partie de ces sépultures au Néolithique final (Courtaud et Dumontier, 2009).

\section{RÉFÉRENCES BIBLIOGRAPHIQUES}

Boule M. (1896) - La grotte d'Isturitz (Basses-Pyrénées), L'Anthropologie, 7, 6, p. 725-726.

Buisson D., Gambier D. (1991) - Façonnage et gravures sur des os humains d'Isturitz (Pyrénées-Atlantiques), Bulletin de la Société préhistorique française, 88, 6, p. 172-177.

Courtaud P., Dumontier P. (2009) - Études préliminaires de la zone funéraire de la salle de Saint-Martin, in C. Normand (dir.), Transition Paléolithique moyen-Paléolithique supérieur et évolution de l'Aurignacien dans la salle de SaintMartin de la grotte d'Isturitz (commune de Saint-Martind'Arberoue). Phase I : la séquence aurignacienne, rapport de fouilles programmées 2009, non publié, déposé au service régional de l'Archéologie d'Aquitaine, étude complémentaire $\mathrm{n}^{\circ} 6$.

Delporte H. (1974) - Le Moustérien d'Isturitz d'après la collection Passemard (musée des Antiquités nationales), Zephyrus, 25, p. 17-42.

Delporte H. (1980-1981) - La collection Saint-Périer et le Paléolithique d'Isturitz : une acquisition prestigieuse, Antiquité nationales, 12-13, p. 20-26.

ESPARZA SAN JUAN X. (1995) - La cueva de Isturitz, su yacimiento y sus relaciones con la Cornisa cantabrica durante el Paleolitico superior, Madrid, UNED, 309 p.

Esparza San Juan X, Mujika Alustiza J. A. (1996) - La cueva de Isturitz en el Pirineo occidental, in $\mathrm{H}$. Delporte et J. Clottes (dir.), Pyrénées préhistoriques, arts et sociétés, actes du $118^{\mathrm{e}}$ Congrès des sociétés historiques et scientifiques (Pau, 25-29 octobre 1993), Paris, CTHS, p. 73-86.

Foucher P. (2004) - Les industries lithiques du complexe Gravettien-Solutréen dans les Pyrénées : technotypologie et circulation des matières siliceuses de part et d'autre de l'axe Pyrénées-Cantabres, thèse de doctorat, université Toulouse 2 - Le Mirail, $334 \mathrm{p}$.

GAMBIER D. (1990-1991) - Les vestiges humains du gisement d'Isturitz (Pyrénées-Atlantiques), étude anthropologique et analyses des traces d'action humaine intentionnelle, Antiquités nationales, 22-23, p. 9-26.

GAmbier D. (1996) - Les pratiques funéraires au Magdalénien dans les Pyrénées françaises, in $\mathrm{H}$. Delporte et J. Clottes (dir.), Pyrénées préhistoriques, arts et sociétés, actes du $118^{\mathrm{e}}$ Congrès des sociétés historiques et scientifiques (Pau, 25-29 octobre 1993), Paris, CTHS, p. 263-277.

Goutas, N. (2004) - Caractérisation et évolution du Gravettien en France par l'analyse techno-économique des industries en matières osseuses, thèse de doctorat, université Paris IPanthéon-Sorbonne, 2 vol., 675 p.

Henry-Gambier D. (2005) - Les hommes du Paléolithique supérieur européen, in B. Vandermeersch, J.-J. Hublin et O. Dutour (dir.), Origine et évolution des populations humaines, Paris, CTHS (Orientations et méthodes, 8), p. 151-176.

Lacarriere J., Goutas N., Normand C., Simonet A. (2011) Vers une redéfinition des occupations gravettiennes de la grotte d'Isturitz (Pyrénées-Atlantiques, France) : révision critique des collections « anciennes » par l'approche intégrée des données lithiques, fauniques et de l'industrie osseuse, in N. Goutas, L. Klaric, D. Pesesse et P. Guillermin (dir.), À la recherche des identités gravettiennes : actualités, questionnements et perspectives, Paris, Société préhistorique française (Mémoire, 52), p. 67-83.

LANGLAIS M. (2010) - Les sociétés magdaléniennes de l'isthme pyrénéen, Paris, CTHS (Documents préhistoriques, 26), $337 \mathrm{p}$.

Mujıka J. A. (1991) - La industria ósea del Paleolítico superior y Epipaleolítico del Pirineo occidental, thèse de doctorat, Universidad de Deusto, Bilbao, 1351 p.

Normand C. (2002) - L'Aurignacien de la salle de SaintMartin (grotte d'Isturitz, commune de Saint-Martin-d'Arberoue, Pyrénées-Atlantiques) : données préliminaires sur l'industrie recueillie lors des campagnes 2000-2002, in F. Bon, J. M. Maillo Fernandez et D. Ortega Cobos (dir.), En torno a los conceptos de Protoauriñaciense, Auriñaciense arcaico, inicial y antiguo. Unidad y variabilidad de los comportamientos tecnológicos de los primeros grupos humanos modernos en el sur de Francia y norte de España, actes de la table ronde (Toulouse, 2003), Madrid, UNED (Espacio, Tiempo y Forma, Serie I-Prehistoria y Arqueologia, 15), p. 145-174.

Normand C. (2005-2006) - Les occupations aurignaciennes de la grotte d'Isturitz (Saint-Martin-d'Arberoue, PyrénéesAtlantiques, France) : synthèse des données actuelles, in «Homenaje a Jesús Altuna », Munibe, 57, 2, p. 119-129.

Normand C., A. De Beaune S., Costamagno S., Diot M.-F., Henry-Gambier D., Goutas N., Laroulandie V., Lenoble A., O'farrell M., Rendu W., Rios Garaizar J., Schwab C., Tarriño Vinagre A., Texier J.-P., White R. (2007) - Nouvelles données sur la séquence aurignacienne de la grotte d'Isturitz (communes d'Isturitz et de Saint-Martin-d'Arberoue, Pyrénées-Atlantiques), in J. Évin 
(dir.), Congrès du centenaire, un siècle de construction $d u$ discours scientifique en Préhistoire, actes du $26^{\mathrm{e}}$ Congrès de la Société préhistorique française (Avignon, 20-25 septembre 2004), Paris, Société préhistorique française, vol. 3, p. $277-293$.

Normand C., Turq A. (2007) - Bilan des recherches 19951998 dans la Grotte d'Isturitz (communes d'Isturitz et de Saint-Martin-d'Arberoue, Pyrénées-Atlantiques), in C. Chauchat (dir.), Préhistoire du bassin de l'Adour : bilans et perspectives, actes du colloque (Saint-Étienne-de-Baigorry, 19 janvier 2002), Saint-Étienne-de-Baigorry, Izpegi, p. 69-101.

Passemard E. (1924) - Les stations paléolithiques du Pays basque et leurs relations avec les terrasses d'alluvions, Bayonne, impr. Bodiou, 218 p.

Passemard E. (1944) - La caverne d'Isturitz en Pays basque, Préhistoire, 9, p. 7-84.

PÉTILlon J.-M. (2004) - Lecture critique de la stratigraphie magdalénienne de la Grande Salle d'Isturitz (PyrénéesAtlantiques), Antiquités nationales, 36, p. 105-131.

PÉTILlon J.-M. (2006) - Des Magdaléniens en armes. Technologie des armatures de projectile en bois de cervidé du Magdalénien supérieur de la grotte d'Isturitz (PyrénéesAtlantiques), Treignes, CEDARC (Artefacts, 10), 302 p.

Pétillon J.-M., Letourneux C., Laroulandie V. (à paraître) - Archéozoologie des collections anciennes : le cas de la faune du Magdalénien supérieur d'Isturitz, in C. Normand (dir.), Les recherches archéologiques dans les grottes d'Isturitz et d'Oxocelhaya de 1912 à nos jours : une synthèse des résultats, actes de la table ronde du cinquantenaire du classement comme monument historique des grottes d'Isturitz et d'Oxocelhaya (Hasparren, 14-15 novembre 2003).

Reimer P. J., Baillie M. G. L., Bard E., Bayliss A., Beck J. W., Blackwell P. G., Bronk Ramsey C., Buck C. E., Burr G. S., Edwards R. L., Friedrich M., Grootes P. M., Guilderson T. P., Hajdas I., Heaton T. J., Hogg A. G., Hughen K. A., Kaiser K. F., Kromer B., Mccormac F. G., Manning S. W., Reimer R. W., Richards D. A., Southon J. R., Talamo S., Turney C. S. M., Van Der Plicht J., Weyhenmeyer C. E. (2009) - IntCal09 and Marine09 Radiocarbon Age Calibration Curves, 0-50,000 Years cal BP, Radiocarbon, 51, 4, p. 1111- 1150.

SAINT-PÉRIER R. DE (1930) - La grotte d'Isturitz, I. Le Magdalénien de la salle Saint-Martin, Paris, Masson (Archives de l'Institut de paléontologie humaine, 7), $124 \mathrm{p}$.

SAINT-PÉRIER R. DE (1936) - La Grotte d'Isturitz, II. Le Magdalénien de la Grande Salle, Paris, Masson (Archives de l'Institut de paléontologie humaine, 17), $138 \mathrm{p}$.

SAINT-PÉRIER R. DE (1947) - Les derniers objets magdaléniens d'Isturitz, L'Anthropologie, 51, p. 393-415.

SAint-Périer R. De, SAINT-Périer S. DE (1952) - La Grotte d'Isturitz, III. Les Solutréens, les Aurignaciens et les Mous- tériens, Paris, Masson (Archives de l'Institut de paléontologie humaine, 25), $264 \mathrm{p}$.

SaINT-Périer S. DE (1965) - Réflexions sur le Paléolithique supérieur d'Isturitz, in E. Ripoll Perello (dir.), Miscelánea en homenaje al Abate Henri Breuil, Barcelone, Diputación Provincial de Barcelona-Instituto de Prehistoria y Arqueología, vol. 2, p. 319-326.

Schwab C. (2002) - Les os à impression et éraillures de la grotte d'Isturitz (Pyrénées-Atlantiques, France), in M. Patou-Mathis, P. Cattelain et D. Ramseyer (dir.), L'industrie osseuse pré- et protohistorique en Europe. Approche technologiques et fonctionnelles, actes du colloque $1.6 \mathrm{du}$ $\mathrm{XIV}^{\mathrm{e}}$ Congrès de l'UISPP (Liège, 2 au 8 septembre 2001), Bulletin du Cercle archéologique Hesbaye-Condroz, 26, p. 9-18.

Simonet A. (2009) - Les Gravettiens des Pyrénées : des armes aux sociétés, thèse de doctorat, université Toulouse 2 Le Mirail, $391 \mathrm{p}$.

Szmidt C., Pétillon, J.-M., Cattelain, P., Normand C., Schwab C. (2009) - Premières dates radiocarbone pour le Magdalénien d'Isturitz (Pyrénées-Atlantiques), Bulletin de la Société préhistorique française, 106, 3, p. 583-601.

Szmidt C., Normand C., Burr G., Hodgins G., Lamotta S. (2010) - AMS ${ }^{14} \mathrm{C}$ dating the Protoaurignacian/Early Aurignacian of Isturitz, France. Implications for Neanderthalmodern humans interaction and the timing of technical and cultural innovations in Europe, Journal of Archeological Science, 37, p. 758-768.

Dominique Henry-Gambier

CNRS, UMR 5199 « PACEA », Anthropologie des populations passées et présentes Université Bordeaux 1

B8, avenue des Facultés, 33405 Talence d.gambier@pacea.u-bordeaux1.fr

Christian NoRMAND UMR 5608 « TRACES » Service régional de l'Archéologie CCE d'Hasparren 54, rue Francis-Jammes, 64240 Hasparren cpjnormand@wanadoo.fr

Jean-Marc PÉtillon CNRS, UMR 5608 «TRACES »

Maison de la recherche Université Toulouse 2 5, allées A.-Machado, 31058 Toulouse cedex 9 petillon@univ-tlse2.fr 